\title{
BMJ Open Association between nut intake and non-alcoholic fatty liver disease risk: a retrospective case-control study in a sample of Chinese Han adults
}

\author{
Bing bing Chen, ${ }^{1}$ Ying Han, ${ }^{2}$ Xinting Pan, ${ }^{1}$ Jianhui Yan, ${ }^{1}$ Wenjuan Liu, ${ }^{1}$ \\ Yangfan Li, ${ }^{1}$ Xu Lin, ${ }^{3}$ Shanghua $\mathrm{Xu},{ }^{4}$ Xian-E Peng ${ }^{1,3}$
}

To cite: Chen Bbing, Han $Y$, Pan $\mathrm{X}$, et al. Association between nut intake and nonalcoholic fatty liver disease risk: a retrospective casecontrol study in a sample of Chinese Han adults. BMJ Open 2019;9:e028961. doi:10.1136/ bmjopen-2019-028961

- Prepublication history for this paper is available online To view these files, please visit the journal online (http://dx.doi. org/10.1136/bmjopen-2019028961).

$\mathrm{BbC}$ and $\mathrm{YH}$ contributed equally.

Received 07 January 2019 Revised 05 August 2019 Accepted 07 August 2019
Check for updates

(C) Author(s) (or their employer(s)) 2019. Re-use permitted under CC BY-NC. No commercial re-use. See rights and permissions. Published by BMJ.

For numbered affiliations see end of article.

Correspondence to

Dr Xian-E Peng;

fmuxe@163.com

\section{ABSTRACT}

Objectives Nut consumption has been associated with a lower risk of type 2 diabetes, metabolic syndrome and insulin resistance. However, its effect on the risk of nonalcoholic fatty liver disease (NAFLD) is unknown. Therefore, we investigated the relationship between nut consumption and NAFLD risk.

Setting and participants We conducted a retrospective case-control study including 534 patients diagnosed with NAFLD and 534 controls matched by sex and age $( \pm 5$ years) from the Affiliated Nanping First Hospital of Fujian Medical University in China.

Main outcome measures Information on dietary intake was collected using a semiquantitative food frequency questionnaire and nut consumption was calculated. Nut consumption was categorised using quartiles based on the distribution of daily nut intake of the controls. Binary logistic regression models were used to estimate ORs and the $95 \%$ Cls for the association between nut consumption and NAFLD risk.

Results After adjusting for potential confounding variables, nut consumption was not associated with NAFLD risk in the overall sample. When the fully adjusted model was stratified by sex, a significant inverse association was found between high nut consumption and NAFLD only among the men in the highest quartile $(0 \mathrm{R}=0.43 ; 95 \% \mathrm{Cl}$ 0.26 to $\left.0.71 ; P_{\text {trend }}=0.01\right)$. The inverse association of nut consumption with NAFLD risk in men remained significant after controlling for other known or suspected risk factors for NAFLD.

Conclusions Diets with a higher intake of nuts may be associated with a decreased risk of NAFLD, particularly in men.

\section{INTRODUCTION}

Non-alcoholic fatty liver disease (NAFLD) is defined as macrovesicular steatosis in $\geq 5 \%$ of hepatocytes, in the absence of a secondary cause, such as alcohol or drug use. NAFLD has become a leading cause of chronic liver disease, with a $25 \%$ prevalence worldwide. ${ }^{1}$ Furthermore, a fatty liver is more prevalent in men compared with women. ${ }^{2}$ The prevalence of NAFLD among the adults in China's

\section{Strengths and limitations of this study}

- In this study, several potential confounding variables such as energy intake and physical activity, were taken into account.

- The study had tried to investigate interactions between nut intake and other risk factors, for which a biologically plausible mechanism may exist.

- This study was a case-control design, thus the causal association between nut intake and non-alcoholic fatty liver disease could not be precisely identified.

- This study was a case-control study, recall bias was inevitable and randomised controlled trial studies are therefore required for more accurate results.

general population is approximately $15 \%$ $(6.3 \%-27.0 \%)$, depending on the population studied. ${ }^{3}$ Many studies suggest that diet and lifestyle may significantly influence the risk of NAFLD. ${ }^{45}$ These studies indicate that type 2 diabetes (T2D), ${ }^{6}$ metabolic syndrome (MetS), obesity, ${ }^{7}$ physical activity and a high-fat $\operatorname{diet}^{4}$ are associated with an increased risk for the development of NAFLD.

Nuts are nutrient-dense foods with complex matrices rich in unsaturated fatty and other bioactive compounds (eg, high-quality vegetable protein, fibre, minerals, tocopherols, phytosterols and phenolic compounds) ${ }^{8}$ The global intake of nuts has increased by $59 \%$ over the past decade. ${ }^{9}$ China's per capita intake of nuts was $2.2 \mathrm{~g} /$ day in 1982 , and it increased to $3.8 \mathrm{~g}$ /day in 2012. ${ }^{10}$ Although they are energy-dense and high in fat, a high intake of nuts has been associated with several health benefits, including reduced risk of cardiovascular disease, ${ }^{11} \mathrm{~T} 2 \mathrm{D},{ }^{12} \mathrm{MetS},{ }^{13}$ and insulin resistance. ${ }^{14}{ }^{15}$ Moreover, nuts have antioxidative effects by decreasing lipid peroxidation and protecting against oxidative DNA damage. ${ }^{16}$ Beneficial health effects 
have been attributed to the macronutrient and micronutrient profiles of nuts. ${ }^{17}$

NAFLD is regarded as the 'hepatic manifestation of MetS'. Few studies have assessed the effects of nut intake on NAFLD, despite previous findings of an inverse correlation between high nut consumption and the risk of T2D and cardiovascular disease. In this study, we analysed the association between nut intake and NAFLD risk and the interactions between nut intake and other established NAFLD risk factors in a large case-control study with a sample of Han adults in China.

\section{METHODS}

\section{Patient and public involvement statement}

Patients and public will not be involved in the development of the research question or in the design of the study. Subjects will receive oral and written information about this study, however, they will not be involved in the recruitment and conduct of the study. After signing informed consent, the participants will be assessed for eligibility and data collection will begin. Eligible subjects will be interviewed face to face by investigators to collect data. In addition, all methods were performed in accordance with the relevant guidelines and regulations.

\section{Study design}

We conducted the retrospective case-control study in a health examination centre at the Affiliated Nanping First Hospital of Fujian Medical University from April 2015 to August 2017. Data were obtained from subjects who underwent routine health examination in the examination centre. Patients newly diagnosed with NAFLD using abdominal ultrasonography in accordance with the 'Guidelines for the diagnosis and management of nonalcoholic fatty liver disease: update 2010' were included in the study. Hepatic ultrasonography examination was performed by trained ultrasonographists who were blinded to the clinical and laboratory data. Hepatic steatosis was diagnosed by characteristic echo patterns according to conventional criteria, such as the evidence of diffuse hyperechogenicity of the liver relative to the kidneys, ultrasound beam attenuation and poor visualisation of intrahepatic structures.

\section{Sample size calculation}

This study is a case-control design, thus we estimate the sample size based on the case-control study formula: ${ }^{18}$

$$
\begin{aligned}
& n=2 \bar{p} \bar{q}\left(z_{\alpha}+z_{\beta}\right)^{2} /\left(p_{1}-p_{0}\right)^{2}, p_{1}=\frac{p_{0} R R}{1+p_{0}(R R-1)}, \\
& \bar{p}=0.5 \times\left(p_{1}+p_{0}\right), \bar{q}=1-\bar{p} .
\end{aligned}
$$

n: sample size; $\alpha$ : probability of type I error; $\beta$ : probability of type II error; p0: exposure rate of control population; RR: risk ratio of exposure factors. By consulting the literature, ${ }^{18}$ we estimate $\mathrm{OR}_{\text {nut intake }}=0.72, \rho_{0}=0.50, \alpha=0.05$, $\beta=0.20$, the calculated sample size was $\mathrm{N}_{\text {case }}=\mathrm{N}_{\text {control }}=609$. Finally 1068 subjects (534 cases and 534 controls) were recruited in this study.

\section{Outcome eligibility of NAFLD cases and controls}

All participants were of Chinese Han ethnicity.The cases were newly diagnosed with NAFLD. The exclusion criteria were as follows: (A) Daily alcohol intake of $>40 \mathrm{~g}$ (men) and $>20 \mathrm{~g}$ (women). (B) A history of other liver diseases including drug-induced liver disease, viral hepatitis, autoimmune hepatitis, total parenteral nutrition and hepatolenticular degeneration. (C) Taking hypolipidaemic or weight reduction drugs. (D) Age $<18$ years or $>70$ years. (E) Non-resident of Nanping. (f) Not of Han ethnicity. Adults who reported extremely abnormal levels of energy intake (2 $511.60 \mathrm{~kJ}(600 \mathrm{kcal})$ or $17581.20 \mathrm{~kJ}(4200 \mathrm{kcal})$ per day for men; $2093 \mathrm{~kJ}$ (500 kcal) or $14651.00 \mathrm{~kJ}(3500$ kcal) per day for women), and those who did not answer 25 or more food-related items or questions about nut intake on the questionnaire, were excluded from the study.

The controls were randomly selected from the same centre during the study period. Their eligibility criteria were identical to those of the cases, except for the requirement of a diagnosis of liver steatosis; they were frequency-matched with cases by age (within 5-year intervals), gender, ethnicity and region of origin.

\section{Ethical considerations}

All subjects who participated in this study provided written informed consent. In addition, all methods were performed in accordance with the relevant guidelines and regulations of the university.

\section{Potential confounders: data measurements and data collection}

Trained interviewers performed a comprehensive medical history on each participant that included eliciting information about their demographic and socioeconomic characteristics (eg, age, gender, education, income, marriage status and history of diabetes, hypertension, hyperlipidaemia), lifestyle habits (eg, smoking, drinking (alcohol), tea drinking and physical activity) and anthropometric assessment (eg, height, body weight and blood pressure). The data were obtained from participants using structured questionnaires during face-to-face interviews.

\section{Exposure: nut consumption}

Information about participants' typical food consumption was collected using a semiquantitative food frequency questionnaire that included 110 food items, which was developed and validated in a sample from southern China. ${ }^{19}$ Participants were asked to estimate the average frequency of consumption of selected foods using the following response options: rarely, <once/month, 1-3 times/month, 1-2 times/week, 3-4 times/week, 5-6 times/week, once/day, twice/day and >twice/day. Nut intake was defined as the consumption of 'peanuts, walnuts, seeds, or other nuts'. Data from a semiquantitative food frequency questionnaire were used to calculate daily nut and energy intake. Nut consumption was 
converted to grams/day by multiplying the food intake frequency by fixed portion sizes. Nutrient intake, such as monounsaturated fatty acids (MUFAs) and polyunsaturated fatty acids (PUFAs) were calculated by multiplying the intake frequency of each food by the nutrient content of the specified portion, and summing the products of all the food items.

\section{Statistical analyses}

Participants' characteristics were analysed using Pearson's $\chi^{2}$ test for categorical variables, and Wilcoxon's rank-sum test and analysis of variance for continuous variables. Nut consumption was categorised by quartiles based on the distribution of daily nut intake by the controls (Q1-Q4). Binary logistic regression models were used to estimate the ORs and their $95 \%$ CIs for the associations between nut consumption and NAFLD risk. To account for the energy content of the nut, our initial model adjusted for total energy intake by using the residuals method. The final model adjusted for potential confounders, including age, income, smoking status, education level and tea-drinking status, occupational status, marital status, body mass index (BMI), physical activity and history of diabetes, hypertension and hyperlipidaemia. In order to reflect the matching protocol, when adjust for age, we entered a term for residual age into the regression analysis. ${ }^{20}$ The final model also adjusted for MUFA and PUFA intake to control for their effects. The selection of covariates for the final model was based on clinical significance and results of previous studies. We tested for linear trends across categories of nut intake by assigning each participant the median value for each category and modelling this value as a continuous variable, consistent with prior studies. ${ }^{21-23}$

We evaluated the influence of nut consumption across strata of other potential confounders, comparing participants in the highest nut consumption category to the lowest nut consumption category (reference). We also analysed the interactions of nut consumption with age, sex, BMI, smoking status, education level, tea drinking status and other factors. We used the medians of the continuous variables to categorise them and evaluate the interactions. The criteria for statistical significance of the likelihood ratio test of interaction effects was $p<0.05$. Statistical analyses were performed using SPSS V.22 (IBM, Armonk, New York, USA). All p values were two-tailed, and $\mathrm{p}<0.05$ was considered statistically significant.

\section{RESULTS}

\section{Baseline characteristics}

Table 1 presents health-related and demographic characteristics of the NAFLD cases and matched controls. Compared with the controls, the NAFLD cases tended to have a higher BMI, higher total intake of energy, higher MUFA and PUFA consumption, and a higher number of participants with a history of hyperlipidaemia among both men and women. Patients with NAFLD had a lower education level and the men engaged in less physical activity than the controls. No statistically significant associations were found for age, occupational status, income, marital status, smoking status, history of diabetes or hypertension between the cases and the controls.

\section{The associations of dietary nut intake with NAFLD}

The participants' nut consumption is summarised in table 2. Among the women, those in the NAFLD group consumed a significantly higher amount of nuts $(6.80 \mathrm{~g} /$ day vs $2.50 \mathrm{~g} /$ day; $\mathrm{p}=0.02$ ) than those in the control group. No statistically significant differences were found between the cases and controls in the sample or among the male participants. In order to adjust for potential confounding factors, quartile distributions of dietary nut consumption among the controls were used to categorise the nut intake of all the participants; the results are shown in table 3. After adjusting for potential confounders, nut consumption was not associated with NAFLD risk among the participants in the sample. When the fully adjusted model was stratified by sex, a significant inverse association was found between nut consumption and NAFLD, but only among the men in the highest quartile $(\mathrm{OR}=0.43$; $95 \%$ CI 0.26 to $\left.0.71 ; P_{\text {trend }}=0.01\right)$.

\section{Stratified analyses}

In the stratified analysis, the inverse association between total nut consumption and NAFLD risk was consistent across strata of age, sex, BMI, education level, income, physical activity, smoking, tea drinking and history of diabetes, hypertension and hyperlipidaemia. In addition to the association between nut consumption and NAFLD in men, we also found the highest nut consumption category was associated with a lower risk of developing NAFLD than the reference $(\mathrm{OR}=0.53 ; 95 \%$ CI 0.31 to 0.93 ) among the participants who engaged in light and moderate physical activity. No significant interactions of total nut consumption and the potentially confounding effects of NAFLD risk factors were identified (figure 1).

\section{DISCUSSION}

In this case-control study, we found a significant inverse relationship between high nut consumption and NAFLD risk among the men, but found either no or unclear associations among the women and the overall sample. Moreover, the associations seemed to be independent of other predictors, including diet and lifestyle factors. The effect of nut consumption remained among men, after controlling for other known or suspected risk factors, for developing NAFLD. Furthermore, no significant interactions between nut consumption and the potential modifying effects of NAFLD risk factors were identified. To the best of our knowledge, this was the first study to assess the association between nut consumption and the risk of NAFLD in a Chinese sample.

Our results are consistent with a previous study conducted in Korea, which found that a low intake of 
Table 1 General characteristics of cases and controls stratified by sex, $n(\%)$

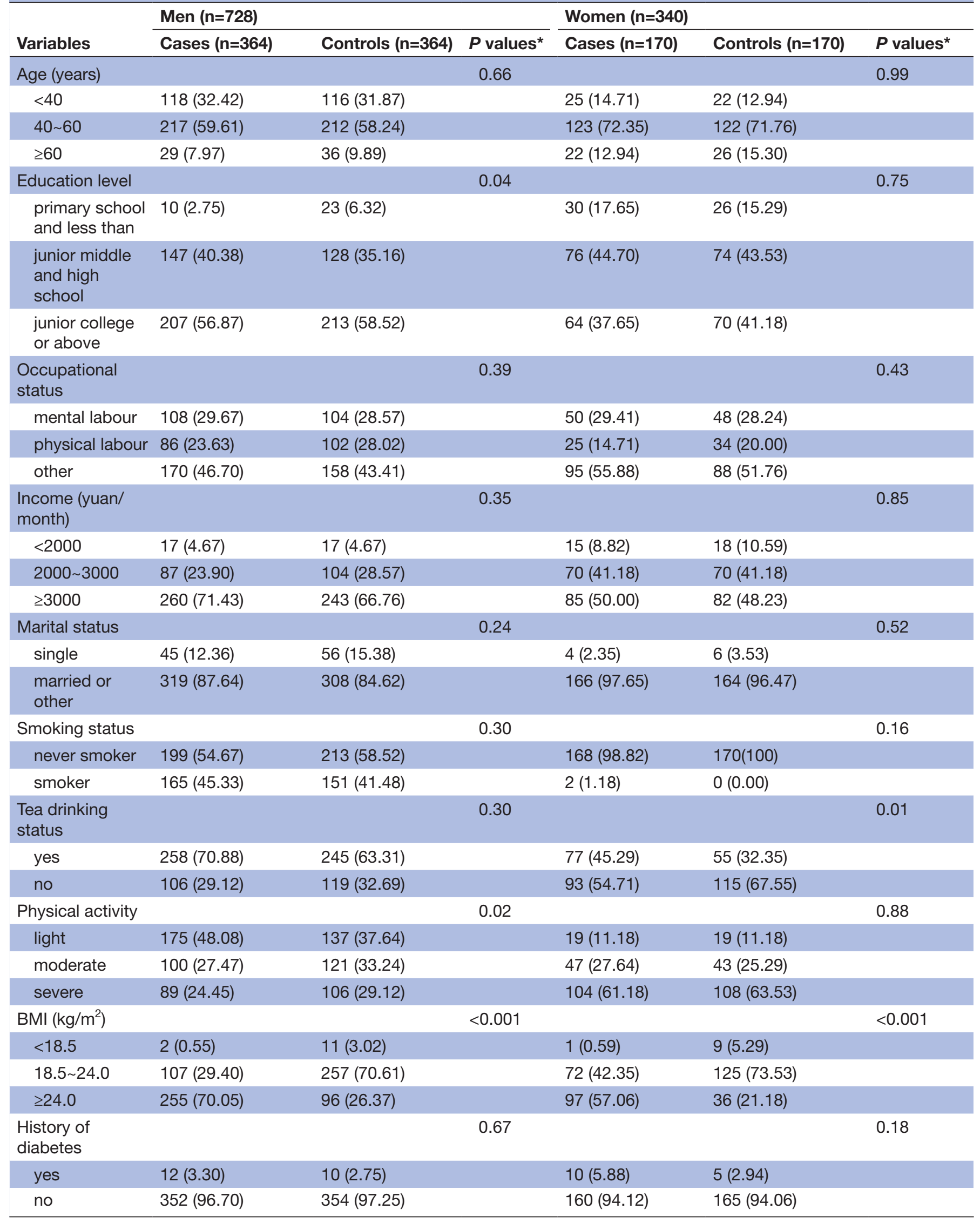


Table 1 Continued

\begin{tabular}{|c|c|c|c|c|c|c|}
\hline \multirow[b]{2}{*}{ Variables } & \multicolumn{3}{|l|}{ Men $(n=728)$} & \multicolumn{3}{|l|}{ Women $(n=340)$} \\
\hline & Cases $(n=364)$ & Controls $(n=364)$ & $P$ values ${ }^{*}$ & Cases $(n=170)$ & Controls $(n=170)$ & $\boldsymbol{P}$ values* \\
\hline $\begin{array}{l}\text { History of } \\
\text { hypertension }\end{array}$ & & & 0.85 & & & 0.63 \\
\hline yes & $15(4.12)$ & $16(4.40)$ & & $8(4.71)$ & $10(5.88)$ & \\
\hline no & 349 (95.88) & 348 (95.60) & & 162 (95.29) & 160 (94.12) & \\
\hline yes & 108 (29.67) & 77 (21.15) & & $51(30.00)$ & $21(12.35)$ & \\
\hline no & $256(70.33)$ & $287(78.85)$ & & $119(70.00)$ & $149(87.65)$ & \\
\hline $\begin{array}{l}\text { MUFA intake, g/ } \\
\text { day† }\end{array}$ & $34.10(28.49-39.65)$ & $\begin{array}{l}31.39(26.55- \\
36.79)\end{array}$ & $<0.001$ & $\begin{array}{l}29.99(26.37- \\
36.37)\end{array}$ & 28.05 (22.40-31.39) & $<0.001$ \\
\hline
\end{tabular}

${ }^{*} P$ values were calculated by using the $\chi^{2}$ test for categorical variables and Wilcoxon rank-sum test and analysis of variance (ANOVA) tests for continues variables.

†Medians (IQRs).

$\ddagger$ Mean (SD).

BMI, body mass index; MUFA, monounsaturated fatty acid; PUFA, polyunsaturated fatty acid.

nuts and seeds $\left(\mathrm{OR}=3.66 ; P_{\text {trend }}=0.007\right)$ was associated with a significantly higher risk of developing NAFLD among male participants, but not among female. ${ }^{24}$ Although another case-control study found an association between a high intake of nuts with a lower likelihood of NAFLD after adjusting for the confounders age, sex, waist circumference and the values of the homoeostasis model assessment of insulin resistance $(\mathrm{OR}=0.61 ; 95 \% \mathrm{CI}$ 0.38 to 0.98 ), the association disappeared after further adjustment for adiponectin and tumor necrosis factor- $\alpha$ $(\mathrm{TNF}-\alpha) \quad(\mathrm{OR}=0.72 ; 95 \% \mathrm{CI} 0.41$ to 1.25$) .{ }^{18}$ However, the study did not examine nut intake separately; therefore, the results cannot be directly compared with those of our study. Although the results of our study suggest nuts may play a protective role in the development of NAFLD in men, a significant association between nut intake and the risk of NAFLD was not found in women. NAFLD has been regarded as the 'hepatic manifestation of MetS'; therefore, our findings are indirectly supported by several studies showing an inverse correlation between high nut intake and the risk of T2D and cardiovascular disease, which share common metabolic parameters with NAFLD. ${ }^{25-27}$ Nevertheless, the relationship between nut intake and NAFLD warrants further exploration, and additional studies are needed to examine gender differences in the association between nut consumption and NAFLD, and its possible mechanisms should be explored.

Several biological pathways have been proposed to explain the association between nut intake and NAFLD risk. Ellagic acid, a natural antioxidant polyphenol found in nuts, can suppress oxidative stress and inflammation ${ }^{28}$ and improve hepatic insulin sensitivity and lipid metabolism. ${ }^{29}$ Vitamin E and selenium in almonds and walnuts, as well as folic acid and resveratrol in pine nuts, have been reported to have beneficial effects on NAFLD. Numerous trials testing the therapeutic value of vitamin $\mathrm{E}$ in NAFLD prevention found that vitamin $\mathrm{E}$ significantly improved liver function and histological changes by significantly reducing aspartate aminotransferase, alanine aminotransferase, steatosis and inflammation in patients with

Table 2 Comparison of daily nut intake between the case and the control

\begin{tabular}{|c|c|c|c|c|c|c|}
\hline Nut intake & Case & & Control & & & \\
\hline (g/day) & Median & Quartile & Median & Quartile & $P$ values* & valuest \\
\hline Total population & 3.15 & $1.46-8.80$ & 2.86 & $1.22-8.98$ & 0.35 & 0.18 \\
\hline Women & 6.80 & $1.75-8.86$ & 2.50 & $1.07-7.84$ & 0.01 & 0.02 \\
\hline
\end{tabular}

${ }^{*} P$ values calculated by using Wilcoxon rank-sum test before adjusting for energy.

$\dagger P$ values calculated by using Wilcoxon rank-sum test after adjusting for energy by using the residual's methods. 


\begin{tabular}{|c|c|c|c|c|c|}
\hline Nut intake & Case $n$ & Control $n$ & Crude OR $(95 \% \mathrm{Cl})$ & Model 1* OR $(95 \% \mathrm{Cl})$ & Model 2* OR $(95 \% \mathrm{Cl})$ \\
\hline Total population $\dagger$ & 534 & 534 & & & \\
\hline Q1 & 125 & 133 & 1 & 1 & 1 \\
\hline Q2 & 112 & 134 & 1.30 (0.93 to 1.81$)$ & 0.89 (0.63 to 1.26$)$ & 0.85 (0.56 to 1.29$)$ \\
\hline Q3 & 159 & 134 & 1.77 (1.29 to 2.44$)$ & 1.26 (0.90 to 1.77$)$ & 1.01 (0.68 to 1.50$)$ \\
\hline Q4 & 138 & 133 & 1.17 (0.81 to 1.68$)$ & $1.10(0.78$ to 1.55$)$ & 0.67 (0.44 to 1.02$)$ \\
\hline$P$ for trend $\ddagger$ & & & 0.46 & 0.45 & 0.07 \\
\hline Men & 364 & 364 & & & \\
\hline Q1 & 106 & 102 & 1 & 1 & 1 \\
\hline Q2 & 91 & 79 & 1.31 (0.88 to 1.94$)$ & 0.90 (0.60 to 1.35$)$ & 0.88 (0.54 to 1.43$)$ \\
\hline Q3 & 74 & 123 & 1.81 (1.24 to 2.65$)$ & 1.73 (1.16 to 2.57$)$ & 1.34 (0.84 to 2.16$)$ \\
\hline Q4 & 93 & 60 & 1.04 (0.66 to 1.63 ) & 0.67 (0.44 to 1.02$)$ & 0.43 (0.26 to 0.71$)$ \\
\hline$P$ for trend $\ddagger$ & & & 0.81 & 0.04 & 0.01 \\
\hline Women & 170 & 170 & & & \\
\hline Q1 & 23 & 27 & 1 & 1 & 1 \\
\hline Q2 & 33 & 43 & 1.28 (0.69 to 2.37 ) & 0.90 (0.44 to 1.85$)$ & 0.84 (0.36 to 1.97$)$ \\
\hline Q3 & 36 & 60 & 1.71 (0.95 to 3.08 ) & 0.70 (0.35 to 1.41$)$ & 0.55 (0.24 to 1.25$)$ \\
\hline Q4 & 78 & 40 & 1.41 (0.76 to 2.64$)$ & 2.29 (1.17 to 4.49$)$ & 1.30 (0.57 to 2.95$)$ \\
\hline$P$ for trend $\neq$ & & & 0.36 & $<0.001$ & 0.12 \\
\hline
\end{tabular}

For the total population: Q1, <1.46 g/day; Q2, 1.46-3.15g/day; Q3, 3.15-8.80 g/day; Q4, $\geq 8.80 \mathrm{~g} /$ day. For men: Q1, $<1.15 \mathrm{~g} /$ day; Q2, 1.15-2.68 g/day; Q3, 2.68-8.43 g/day; Q4, >8.43 g/day. For women: Q1, <1.75g/day; Q2, 1.75-6.80 g/day; Q3, 6.80$8.86 \mathrm{~g} / \mathrm{day} ; \mathrm{Q} 4,28.86 \mathrm{~g} /$ day.

${ }^{*}$ Model 1: Adjusted for total energy intake. Model 2: Adjusted further for age, income, smoking status, educational level, tea-drinking status, occupationanl status, marital status, body mass index, physical activity, the history of diabetes, hypertension and hyperlipidaemia, MUFA and PUFA intake.

†Further adiusted for sex.

$\ddagger P$ for trend: Trend across intake levels with the categorical median.

MUFA, monounsaturated fatty acid; NAFLD, non-alcoholic fatty liver disease; PUFA, polyunsaturated fatty acid.

NAFLD/non-alcoholic steatohepatitis (NASH). ${ }^{30}$ Selenium is an antioxidant agent, and selenium deficiency is associated with insulin resistance in patients with chronic liver disease. ${ }^{31}$ Folate plays an essential role in lipid metabolism and folic acid supplementation can attenuate steatosis and improve oxidative stress in rodent models of NAFLD. Folate can also blunt the increase of inflammatory cytokines secreted by immune cells. ${ }^{32}$ Resveratrol has antioxidant, antiapoptotic and anti-inflammatory properties in patients with NAFLD. ${ }^{33}$ Nuts, which are known to be rich in unsaturated fatty acids, are especially rich in n-3 PUFAs, a source of $\alpha$-linolenic acid, which lowers low density lipoprotein (LDL) cholesterol, ${ }^{34}$ and has been found to have beneficial effects on NAFLD. ${ }^{35}$ Each type of nut has many nutrients and phytochemicals that may be beneficial to health, and it is likely that unknown salubrious effects of nuts may be related to NAFLD prevention. Moreover, many studies have shown a beneficial association between high nut intake and decreased risk of obesity, ${ }^{36} \mathrm{~T}^{2} \mathrm{D}^{37}$ and MetS, ${ }^{38}$ which are risk factors for NAFLD.
Our study has both strengths and limitations. The first strength is the large sample size, which may have reduced type II errors. Second, the collection of data using faceto-face interviews and a semiquantitative food frequency questionnaire, which elicited extensive information on anthropometrics and lifestyle factors, allowed us to adjust for confounding factors. Finally, the study tried to investigate interactions between nut intake and other risk factors, for which a biologically plausible mechanism may exist. Nonetheless, several limitations should be considered when interpreting the study's findings. First, although we accounted for a wide range of socioeconomic characteristics and lifestyle factors, we cannot exclude the effects of unknown or poorly measured confounding variables or residual confounding attributable to other dietary/lifestyle factors, which might have influenced the observed associations. However, the associations persisted even after controlling for known and suspected predictors of NAFLD. Second, the intake of nuts was assessed by asking one question; therefore, we could not investigate the effects of different types of nuts, such as peanuts and walnuts, which contain different amounts 


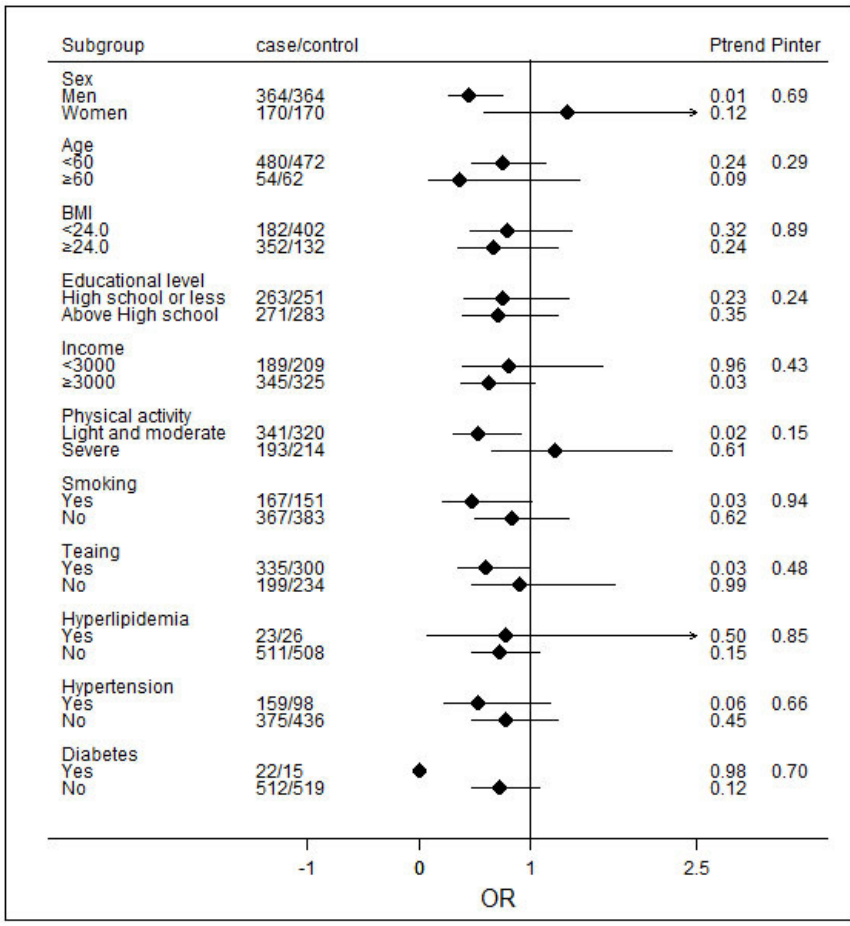

Figure 1 ORs and 95\% Cls for non-alcoholic fatty liver disease (NAFLD) across the strata of various factors. The forest plot represents the ORs of the comparison of the lowest nut intake category versus the highest nut-intake category, adjusting for age, income, smoking status, educational level, tea-drinking status, occupational status, marital status, body mass index (BMI), physical activity, history of diabetes, hypertension and hyperlipidaemia, total energy intake, and monounsaturated fatty acid (MUFA) and polyunsaturated fatty acid (PUFA) intake. $P$ values are twotailed; $P_{\text {inter }}$ indicates $P$ for the interaction between strata and nut intake; $P_{\text {trend }}$ indicates $P$ for the trend across levels of nut intake.

of energy, fat content and other nutrients. ${ }^{39}$ Third, categorisation of the intake of nuts in quartiles may lead to bias and inefficiency. Fourth, we did not consider manufacturing methods (eg, raw, roasted or boiled) or extra ingredients (eg, sugar, salt, seasoning). Different preparation methods before and after roasting, the amount of time roasted and the temperature used can affect the nutrient composition and ingredients in the nuts. ${ }^{40} 41$ What's more, the item 'nuts' in the food frequency questionnaire (FFQ) used in this study cannot represent all nuts, because it only included peanuts, walnuts, seeds. For this reason, direct comparisons with the results of other studies may be difficult. Fifth, because we used a case-control study design, recall bias is an inherent limitation. People who are health-conscious may over-report or under-report some food items. However, the protective effect of nuts on NAFLD was not generally known at the time of the survey, and thus, should be unrelated to recall bias. In addition, the stratified analyses was not corrected for multiple testing, which may inflate the risk for type I error. Sixth, NAFLD is a spectrum of diseases ranging from essentially benign simple steatosis (fatty infiltration of the liver) to the more severe form NASH (fat with inflammation and/or fibrosis). In the present study, the data about the severity of NAFLD were absent, therefore we did not analysis the association between nut intake and the the severity of NAFLD. Seventh, lack of interactions association between nut intake and other risk factors may be due to lack of power, and a small interactive effect on NAFLD cannot be ruled out. Finally, due to the small number of participants in the highest nut intake category, we cannot rule out the possibility that some of our results are due to chance. However, the associations were consistent in the analyses stratified by some of the other factors, which reduces the likelihood of chance findings. In these stratified analyses, the statistical power needed to detect differences was limited by the sample size, and such analyses should be considered exploratory.

Our study had a retrospective design, which is lower on the evidence hierarchy than prospective studies. Nevertheless, case-control studies can provide evidence supporting the general relationship between diet and NAFLD, as there currently are no available prospective studies.

In conclusion, this case-control study indicated that high nut intake was associated with a significantly reduced risk of NAFLD among Han men in China. However, no relationship was found between total nut intake and NAFLD risk among the Han women. Our data contribute to the growing evidence showing that a relatively simple prevention strategy of incorporating a modest amount of nuts in the diet may contribute to maintaining good health at both the individual and population levels.

\section{Author affiliations}

${ }^{1}$ Department of Epidemiology and Health Statistics, Fujian Provincial Key Laboratory of Environment Factors and Cancer, School of Public Health, Fujian Medical University, Fuzhou, China

${ }^{2}$ Fujian Hypertension Research Institute, VIP ward, The First Affiliated Hospital of Fujian Medical University, Fuzhou, , China

${ }^{3}$ Key Laboratory of Ministry of Education for Gastrointestinal Cancer, Fujian Medical University, Fujian, China

${ }^{4}$ Department of Cardiology, Affiliated Nanping First Hospital, Fujian Medical University, Nanping, China

Acknowledgements The authors thank all study participants for their cooperation The authors also thank their staff for recruiting participants and for their technical assistance.

Contributors $\mathrm{BbC}$ and $\mathrm{YH}$ are joint first authors. $\mathrm{X}$-EP obtained funding. $\mathrm{X}$-EP, $\mathrm{XL}$ and $\mathrm{SX}$ designed the study. XP, JY,YL and WL collected the data. $\mathrm{BbC}$ and YH were involved in data cleaning and verification. $\mathrm{BbC}$ and $\mathrm{XP}$ analysed the data. $\mathrm{BbC}$ and YH drafted the manuscript. X-EP, YL and JY contributed to the interpretation of the results and critical revision of the manuscript for important intellectual content and approved the final version of the manuscript. All authors have read and approved the final manuscript.

Funding This work was supported by the National Natural Science Foundation of China (grant number 81473047), the National Natural Science Foundation of Fujian Province (grant number 2019J01316), and the Joint Innovation Project of Science and Technology of Fujian Province (grant number 2017Y9104).

Competing interests None declared.

Ethics approval Local ethics committees of Fujian Medical University (ethics number 2014096).

Provenance and peer review Not commissioned; externally peer reviewed. 
Data availability statement Extra data can be accessed via the Dryad data repository at http://datadryad.org/ with the doi:10.5061/dryad.8nn2j46.

Open access This is an open access article distributed in accordance with the Creative Commons Attribution Non Commercial (CC BY-NC 4.0) license, which permits others to distribute, remix, adapt, build upon this work non-commercially, and license their derivative works on different terms, provided the original work is properly cited, appropriate credit is given, any changes made indicated, and the use is non-commercial. See: http://creativecommons.org/licenses/by-nc/4.0/.

\section{REFERENCES}

1. Maurice J, Manousou P. Non-Alcoholic fatty liver disease. Clin Med 2018;18:245-50.

2. Rietman A, Sluik D, Feskens EJM, et al. Associations between dietary factors and markers of NAFLD in a general Dutch adult population. Eur J Clin Nutr 2018;72:117-23.

3. Fan J-G. Epidemiology of alcoholic and nonalcoholic fatty liver disease in China. J Gastroenterol Hepatol 2013;28(Suppl. 1):11-17.

4. Katsagoni CN, Georgoulis M, Papatheodoridis GV, et al. Effects of lifestyle interventions on clinical characteristics of patients with non-alcoholic fatty liver disease: a meta-analysis. Metabolism 2017;68:119-32.

5. Zelber-Sagi S, Salomone F, Mlynarsky L. The Mediterranean dietary pattern as the diet of choice for non-alcoholic fatty liver disease: evidence and plausible mechanisms. Liver Int 2017;37:936-49.

6. Kosmidou M, Milionis $\mathrm{H}$. Diabetes mellitus and non-alcoholic fatty liver disease: the thread of Ariadne. Minerva Endocrinol 2017;42:109-21.

7. Petrović G, Bjelaković G, Benedeto-Stojanov D, et al. Obesity and metabolic syndrome as risk factors for the development of non-alcoholic fatty liver disease as diagnosed by ultrasound. VSP 2016;73:910-20.

8. Ros E. Health benefits of nut consumption. Nutrients 2010;2:652-82.

9. Lee J, Shin $\mathrm{A}, \mathrm{Oh} \mathrm{JH}$, et al. The relationship between nut intake and risk of colorectal cancer: a case control study. Nutr J 2018;17:37.

10. Lahong J, Dongmei $Y$, Xiaoli $X$. Status and variation trend of nut intake among Chinese residents,2010-2012. Chin J Public Health 2017;33:916-8.

11. Larsson SC, Drca N, Björck M, et al. Nut consumption and incidence of seven cardiovascular diseases. Heart 2018;104:1615-20.

12. Asghari G, Ghorbani Z, Mirmiran P, et al. Nut consumption is associated with lower incidence of type 2 diabetes: the Tehran lipid and glucose study. Diabetes Metab 2017;43:18-24.

13. O'Neil CE, Fulgoni VL, Nicklas TA. Tree nut consumption is associated with better adiposity measures and cardiovascular and metabolic syndrome health risk factors in U.S. adults: NHANES 2005-2010. Nutr J 2015;14:64.

14. Casas-Agustench P, López-Uriarte P, Bulló M, et al. Effects of one serving of mixed nuts on serum lipids, insulin resistance and inflammatory markers in patients with the metabolic syndrome. Nutr Metab Cardiovasc Dis 2011;21:126-35

15. Hernández-Alonso $P$, Salas-Salvadó J, Baldrich-Mora M, et al. Beneficial effect of pistachio consumption on glucose metabolism, insulin resistance, inflammation, and related metabolic risk markers: a randomized clinical trial. Diabetes Care 2014;37:3098-105.

16. Grosso G, Yang J, Marventano S, et al. Nut consumption on all-cause, cardiovascular, and cancer mortality risk: a systematic review and meta-analysis of epidemiologic studies. Am J Clin Nutr 2015;101:783-93.

17. Dreher ML, Maher CV, Kearney $P$. The traditional and emerging role of nuts in healthful diets. Nutr Rev 1996;54:241-5.

18. Katsagoni CN, Georgoulis M, Papatheodoridis GV, et al. Associations between lifestyle characteristics and the presence of nonalcoholic fatty liver disease: a Case-Control study. Metab Syndr Relat Disord 2017;15:72-9.

19. Ke L, Toshiro T, Fengyan S, et al. Relative validity of a semiquantitative food frequency questionnaire versus 3 day weighed diet records in middle-aged inhabitants in Chaoshan area, China. Asian Pac J Cancer Prev 2005;6:376-81.
20. Mansournia MA, Jewell NP, Greenland S, et al. Misconceptions, and recommendations. Eur J Epidemiol 2018;33:5-14.

21. Fadelu T, Zhang S, Niedzwiecki D, et al. Nut consumption and survival in patients with stage III colon cancer: results from CALGB 89803 (Alliance). J Clin Oncol 2018;36:1112-20.

22. Fuchs MA, Sato K, Niedzwiecki D, et al. Sugar-Sweetened beverage intake and cancer recurrence and survival in CALGB 89803 (Alliance). PLoS One 2014;9:e99816.

23. Meyerhardt JA, Sato K, Niedzwiecki D, et al. Dietary glycemic load and cancer recurrence and survival in patients with stage III colon cancer: findings from CALGB 89803. J Natl Cancer Inst 2012;104:1702-11.

24. Han JM, Jo AN, Lee SM, et al. Associations between intakes of individual nutrients or whole food groups and non-alcoholic fatty liver disease among Korean adults. J Gastroenterol Hepatol 2014;29:1265-72.

25. Edens MA, Kuipers F, Stolk RP. Non-Alcoholic fatty liver disease is associated with cardiovascular disease risk markers. Obes Rev 2009;10:412-9.

26. Lonardo A, Ballestri S, Guaraldi G, et al. Fatty liver is associated with an increased risk of diabetes and cardiovascular disease - Evidence from three different disease models: NAFLD, HCV and HIV. WJG 2016;22:9674-93.

27. Gastaldelli A. Insulin resistance and reduced metabolic flexibility: cause or consequence of NAFLD? Clin Sci 2017;131:2701-4.

28. Panchal SK, Ward L, Brown L. Ellagic acid attenuates highcarbohydrate, high-fat diet-induced metabolic syndrome in rats. Eur J Nutr 2013;52:559-68.

29. Polce S, Burke C, França L, et al. Ellagic acid alleviates hepatic oxidative stress and insulin resistance in diabetic female rats. Nutrients 2018;10:531-15.

30. Sato K, Gosho M, Yamamoto T, et al. Vitamin E has a beneficial effect on nonalcoholic fatty liver disease: a meta-analysis of randomized controlled trials. Nutrition 2015;31:923-30.

31. Himoto T, Yoneyama $\mathrm{H}$, Kurokohchi $\mathrm{K}$, et al. Selenium deficiency is associated with insulin resistance in patients with hepatitis $C$ virusrelated chronic liver disease. Nutr Res 2011;31:829-35.

32. Sid V, Siow YL, O K. Role of folate in nonalcoholic fatty liver disease. Can J Physiol Pharmacol 2017;95:1141-8.

33. Hajighasem A, Farzanegi P, Mazaheri Z. Effects of combined therapy with resveratrol, continuous and interval exercises on apoptosis, oxidative stress, and inflammatory biomarkers in the liver of old rats with non-alcoholic fatty liver disease. Arch Physiol Biochem 2018:1-8.

34. Jeznach-Steinhagen A, Ostrowska J, Czerwonogrodzka-Senczyna A et al. Dietetary recommendation for non-alcoholic fatty liver disease]. Pol Merkur Lekarski 2017;43:281-6.

35. Musa-Veloso K, Venditti C, Lee H, et al. Systematic review and meta-analysis of controlled intervention studies on the effectiveness of long-chain omega-3 fatty acids in patients with nonalcoholic fatty liver disease. Nutr Rev 2018:1-22.

36. Mohammadifard N, Yazdekhasti N, Stangl Gl, et al. Inverse association between the frequency of nut consumption and obesity among Iranian population: Isfahan healthy heart program. Eur J Nutr 2015:54:925-31.

37. Hernández-Alonso P, Camacho-Barcia L, Bulló M, et al. Nuts and dried fruits: an update of their beneficial effects on type 2 diabetes. Nutrients 2017;9:673.

38. Hosseinpour-Niazi S, Hosseini S, Mirmiran P, et al. Prospective study of nut consumption and incidence of metabolic syndrome: Tehran lipid and glucose study. Nutrients 2017;9:1056.

39. Wu L, Wang Z, Zhu J, et al. Nut consumption and risk of cancer and type 2 diabetes: a systematic review and meta-analysis. Nutr Rev 2015;73:409-25.

40. Schlörmann W, Birringer M, Böhm V, et al. Influence of roasting conditions on health-related compounds in different nuts. Food Chem 2015;180:77-85.

41. Hosseini Bai S, Darby I, Nevenimo T, et al. Effects of roasting on kernel peroxide value, free fatty acid, fatty acid composition and crude protein content. PLoS One 2017;12:e0184279. 